\title{
Clinical features and associations of 560 cases of motor neuron disease
}

\author{
Ting-Ming Li, Eva Alberman, Michael Swash
}

\begin{abstract}
In 560 cases of motor neuron disease, studied retrospectively from their casenotes in three teaching centres, the age at onset ranged from 13 to 87 years (mean 56 years), and the mean duration of illness until death was 2.6 years. In the subgroup of the disease presenting with progressive bulbar palsy presenting after age 59 years, there was a previously unrecognised excess of females sufficient to equalise the sex ratio of incidence of the disease in this age group. No potentially causative clinical associations emerged; no relation was noted between occupational exposure to leather products, trauma or surgical procedures and the disease. There was a trend for patients with motor neuron disease to give a history of abstention from alcohol.

The cause of motor neuron disease is unknown, but epidemiological studies are important in studying new approaches to this inexorably progressive and fatal disease. In all such studies it is important to assess the accuracy of diagnosis, ${ }^{12}$ as well as evaluating the clinical patterns of the disease so that possible associations that might prove important in assessing new notions of causation can be tested. We have reviewed the clinical data on 560 consecutive patients investigated at three neurological centres in the United Kingdom and report our observations here.
\end{abstract}

\section{Methods}

Patients and controls

The medical records of patients diagnosed with motor neuron disease at The London Hospital, London between 1965-82, at the Radcliffe Infirmary, Oxford between 197684, and at the National Hospital for Nervous Diseases, London between 1976-84 were reviewed. Cases were ascertained from the diagnostic index at each of these hospitals using the ICD codes 356.3 (1964-69), 348.9 (1970$78)$ and $335 \cdot 2(1977-84)$. In these three centres the diagnosis was established in each case only after full clinical evaluation and investigation. The latter included haematological and liver function tests, and radiological studies and, in most cases cerebrospinal fluid (CSF) examination and electrophysiological studies.

In some, muscle biopsy and necropsy studies were available. The specificity of these diagnostic procedures in establishing the diag- nosis of motor neuron disease from multiple sclerosis, cervical spondylosis with myelopathy and stroke, diseases with which motor neuron disease may easily be confused, has been studied previously using a discriminant analysis procedure in 362 of 378 cases. ${ }^{1}$ This showed that $96 \%$ of these cases were correctly classified so that, for these hospitals, there was good agreement in the diagnostic criteria in use. The medical and occupational backgrounds of these patients were compared with those of 220 control patients, taken from the case records of the same three hospitals for the years 1981-84. These control patients suffered from Parkinson's disease, cervical spondylosis, or multiple sclerosis, and had been used as controls in our previous study of the differential diagnosis of motor neuron disease from other neurological conditions. ${ }^{1}$

\section{Sex and age of onset}

Of the 560 patients with motor neuron disease $342(61 \%)$ were male, a sex ratio of $1 \cdot 57: 1$. There was a marked male excess in patients presenting below the age of 60 years, but the sex distribution in patients presenting after this age was nearly equal (fig 1). This change in sex ratio with increasing age was accounted for by the preponderance of women presenting with bulbar symptoms in the older age ranges (fig 2). The mean age of onset for men was 54.6 years (SD 12.5 years) and for women 58 years (SD 11.4 years). In the whole group of 560 patients the mean age of onset was 56 years (range 13-87 years), although $71 \%$ reported the onset of symptoms between age 46 and 66 years (fig 1 ).

\section{Age at death and duration of illness}

The age at death was recorded for only 125 of the 560 cases. In these patients the mean age at death was 57.4 years (SD 12.9 years), and the mean duration of illness was 2.6 years (SD 2.4 years). The age at death ranged from 22 88 years.

\section{Symptoms and signs at presentation}

The majority of patients $(63 \%)$ reported muscle weakness as the first symptom, $23 \%$ reported difficulty in speaking, $16 \%$ difficulty in walking and $11 \%$ muscle wasting. The disease started in the upper limbs in $44 \%$, in the lower limbs in $37 \%$, and in the bulbar muscles in $19 \%$. The latter presentation was especially a feature of older women with motor neuron disease (fig 2). On examination, muscle weakness was noted in the upper limbs in $74 \%$ of the patients, and in the lower limbs 


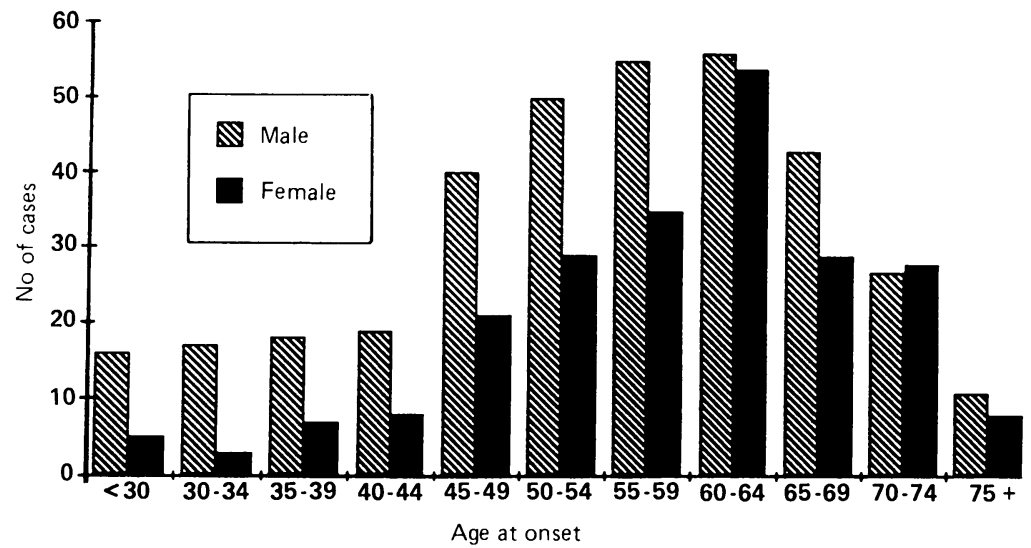

Figure 1 Sex and age distribution of 560 cases of motor neuron disease at presentation.

in $60 \%$. Muscle wasting was found in the upper limbs in $66 \%$, and in the legs in $40 \%$ of patients. Fasciculation was recorded in the upper limbs in $65 \%$, in the lower limbs in $50 \%$, and in the tongue in $37 \%$. The plantar responses were extensor in $47 \%$ of patients. Dysarthria was recorded in $50 \%$, and dysphagia in $41 \%$. Sensory abnormalities were noted in only $2.5 \%$ of the cases, and these had other explanations.

\section{Previous medical history}

There was a history of a surgical operation, ranging from tonsillectomy to hysterectomy, in $39 \%$ of patients with motor neuron disease. A crude comparison of the patients with motor neuron disease and the controls showed a similar rate for surgical procedures, although the mean age of the control subjects was less than that of the patients with motor neuron disease. Analysis of the prevalence of surgical operations in different age groups showed no significant trends or differences from the control patients. Only $0.9 \%$ of patients with motor neuron disease, and $0.5 \%$ of controls reported a history of poliomyelitis. There were no differences in the incidence of other medical conditions between the patients with motor neuron disease and the controls apart from Parkinson's disease, cervical spondylosis and multiple sclerosis, the diagnostic criteria by which these control patients were selected.

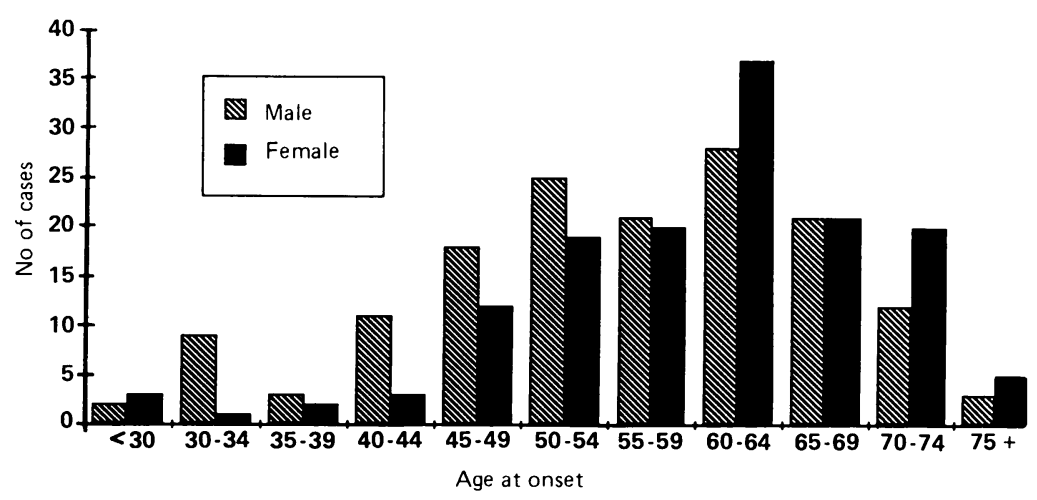

Figure 2 Sex and age distribution of patients with motor neuron disease presenting with progressive bulbar palsy. There is an excess of females in the patients presenting after the age of 59 years.
Tobacco and alcohol usage

Past and present smoking habits in the patients with motor neuron disease showed no differences from the controls, although associations with age and male sex were evident. There was an overall association between motor neuron disease and past or present abstention from alcohol that was significant after adjustment for age and sex $(p=0.0055)$. Thirty six per cent of male and $59 \%$ of female patients with motor neuron disease had never drunk alcohol, compared with $25 \%$ and $44 \%$ respectively of the controls.

\section{Occupational history}

Data on occupation was not recorded in 22 of the 560 patients with motor neuron disease, and in 21 of the 220 control cases. One hundred and twenty nine ( $43 \%)$ of the 218 women in the motor neuron disease group, and 43 $(20 \%)$ of the 114 women in the control group were classified as "housewives", slightly more than would have been predicted from the overall sex ratio. Eighteen per cent of the motor neuron disease patients and $12 \%$ of the controls were in clerical occupations, and 13\% and $10 \%$ respectively were in administrative and executive occupations. There was no difference in the proportions of people engaged in work with leather, leather products, printing or book-binding. No other significant differences between the occupations of patients and controls were noted.

\section{Discussion}

The presenting clinical features in patients with motor neuron disease have been extensively studied. ${ }^{3-13}$ Kurland $^{14}$ noted that the sex ratio was about $1 \cdot 5: 1$, reflecting the preponderance of men in most reported series, although ratios as high as $2 \cdot 5: 1^{5}$ or even $6 \cdot 2: 1$ in India where a different clinical form of the disease seems to exist, ${ }^{15}$ have been noted. In the present series the overall sex ratio was $1 \cdot 57: 1$, but for patients older than 60 years it was equal, an observation suggesting that the sex ratios in previous reports may have been influenced by the age ranges of the patients forming the data bases of these studies. In the earlier reports the mean age at onset was in the sixth decade, as in our series of patients, in all except the cases reported from India. ${ }^{15}$ In all these reported cases there was an approximately equal frequency of origin of the disease in the arms or legs, usually with a smaller proportion beginning in the bulbar musculature.

In our cases the disease started in the upper limbs in $44 \%$, in the lower limbs in $37 \%$, and in the bulbar muscles in $19 \%$. An origin in the bulbar muscles was more likely in older women but, in men bulbar onset tended to occur at a slightly younger age than in women. These age-related features are difficult to explain by the well-documented greater incidence of disease among older men, compared with older women, and seem to indicate 
a relatively specific and unique feature of the disease.

Fasciculation was noted in $62 \%$ of our cases at some stage in the disease, although only $0.8 \%$ of patients reported this phenomenon at presentation. The mean duration of the disease in our study was similar to that reported in other surveys. Review of the case-notes of these 560 patients has failed to reveal any specific life events unique to patients with motor neuron disease that might be regarded as having a causal influence. This included all the items previously reported as having a possible causative relationship in the disease, especially a history of exposure to lead, ${ }^{16}$ trauma, ${ }^{17} 18$ poliomyelitis, ${ }^{19} 20$ and crude operation rates. ${ }^{21} 22$ Martyn et al ${ }^{20}$ noted a statistical relation between the past epidemiology of poliomyelitis and the incidence of motor neuron disease some decades later; our data does not address this notion.

Smoking is positively associated with a number of common and serious diseases, but in Parkinson's disease there is a negative association with smoking. ${ }^{23} 24$ In our patients the prevalence of smoking was similar among patients with motor neuron disease and controls, but less than in the general population as reported in the General Household Survey, ${ }^{25}$ which showed that between 1972-80 smokers made up $50-64 \%$ of men and $37-$ $41 \%$ of women in the general population. In our patients smoking showed an association with sex and age, but no independent association with motor neuron disease $(p=0 \cdot 26)$.

Alcohol use, on the other hand, showed an overall negative association with motor neuron disease $(p=0.0055)$ compared with the controls. Our data, like those of Martyn et $a l,{ }^{20}$ do not confirm the suggestion made by Hawkes et al ${ }^{26}$ that motor neuron disease might be positively associated with occupational exposure to leather goods. No new epidemiological associations that might be important in pathogenesis have been revealed by this retrospective study, although several previously suggested associations have not been confirmed.

We thank the Motor Neurone Disease Association and the Wellcome Trust, for financial support. The staff of The National Hospital for Nervous Diseases, Queen Square, and the Radcliffe Infirmary, Oxford, kindly allowed us access to their case records.

1 Li T-M, Day SM, Alberman E, Swash M. Differential diagnosis of motor neuron disease from other conditions. Lancet 1986;ii:731-3.

2 Li T-M, Swash M, Alberman E, Day SJ. Diagnosis of motor neuron disease; a study of criteria, certainty and consistency in neurologists in three countries. (Submitted).

3 Friedman AP, Freedman D. Amyotrophic lateral sclerosis. J Nerv Men Dis 1950;111:1-18.

4 Muller R. Progressive motor neuron disease in adults; a clinical study with special reference to the course of the disease. Acta Psychiatr Scand 1952;27:137-56.

5 Mackay RP. Course and prognosis in amyotrophic lateral sclerosis. Arch Neurol (Chicago) 1963;8:117-27.

6 Vejiajiva A, Foster JB, Miller H. Motor neurone disease; a clinical study. J Neurol Sci 1967;4:299-314.

7 Cendrowski W, Wender $M$, Owsianowski $M$. Analyse epidemiologique de la sclerose laterale amyotrophique sur la territorie de la Grande-Pologne. Acta Neurol Scand 1970;46:607-17.

8 Muller-Jensen A, Bernhardt W. Unsere Erfahrungen bei der Myatrophischen Lateralsklerose. Nervenarzt 1973;44: 143-9.

9 Jokelainen M. Amyotrophic lateral sclerosis in Finland; 1. an epidemiological study; 2 . clinical characteristics. Acta Neurol Scand 1977;56:185-93, 194-204.

10 Rosen AD. Amyotrophic lateral sclerosis; clinical features and prognosis. Arch Neurol (Chicago). 1978;35:638-42.

11 David P, Lo-Manaco M, Palieri G, et al. Clinical features of amyotrophic lateral sclerosis. Italian Journal of Neurological Sciences 1981;2:113-7.

12 Mortara P, Bardelli D, Leone M, Schiffer D. Prognosis and clinical varieties of amyotrophic lateral sclerosis disease. Italian Journal of Neurological Science 1981;2:237-42.

13 Gubbay SS, Kahana E, Zilber N, Cooper G, Pintov S, Leibowitz Y. Amyotrophic lateral sclerosis; a study of its presentation and prognosis. J Neurol 1985;232:295-300.

14 Kurland LT. Epidemiological investigations of amyotrophic lateral sclerosis. Proc Mayo Clinic 1957;32:449-62.

15 Rai B, Jolly SS. Motor neurone disease; a clinical study. $J$ Indian Med Assoc 1971;57:315-8.

16 Campbell AMG, Williams ER, Barltrop D. Motor neurone disease and exposure to lead. J Neurol Psychiatry 1970;33:877-85.

17 Kondo K, Tsubaki T. Case control studies of motor neuron disease. Arch Neurol 1981;38:220-6.

18 Kurtzke JF, Beebe GW. Epidemiology of amyotrophic lateral sclerosis. 1; a case control comparison based on ALS deaths. Neurology 1980;30:453-62.

19 Poskanzer DC, Cantor HM, Kaplan GS. The frequency of preceding poliomyelitis in amyotrophic lateral sclerosis. In: Norris FH, Kurland LT, eds. Motor neuron disease. In: Norris FH, Kurland LT, eds. Motor neuro

20 Martyn CN, Barker DJP, Osmond C. Motor neurone disease and past poliomyelitis in England and Wales. Lancet 1988; i: 1319-22.

21 Ask-Upmark E. Precipitating factors in the pathogenesis of amyotrophic lateral sclerosis. Acta Med Scand 1961; 170:717-23.

22 Koch MJ, Hoffman P, Brody JA, Edgar AH. Neurologic disorders following surgery for peptic ulcer disease. Arch Neurol 1975;32:206-7.

23 Kessler II, Diamond Ki. Epidemiologic studies in Parkinson's disease; 1. smoking and Parkinson's disease. Am J Epidemiol 1971;94:16-25.

24 Godwin-Austen RB, Lee PN, Marmot MG, Stern GM. Smoking and Parkinson's disease. J Neurol, Neurosurg Smoking and Parkinson's
Psychiatry 1982;45:577-82.

25 General Household Surveys (1972-1980). London. HMSO, (Social Survey Division of the Office of Population Censuses and Statistics).

26 Hawkes $\mathrm{CH}$, Fox AJ. Motor neuron disease in leather workers. Lancet $1981 ; \mathbf{i}: 507$. 